Case

\title{
Apico-Aortic Conduit for Aortic Stenosis in a Patient with Chronic Thoracic Wall Infection
}

\author{
Manabu Shiraishi, MD, Atsushi Yamaguchi, MD, PhD, and Hideo Adachi, MD, PhD
}

\begin{abstract}
We report a case of an apico-aortic bioprothesis-valved conduit for a 70-year-old-woman with symptomatic, severe aortic stenosis and severe calcification of the ascending aorta. She had a history of mastectomy and radiation therapy for breast cancer and was suffering from radiodermatitis and chronic thoracic wall infection. Transthoracic echocardiography showed severe aortic valve stenosis with heavy calcification and high aortic valve pressure gradients. In patients with a chronically infected thoracic wall, median sternotomy is considered to be a high risk procedure, resulting in postoperative mediastiniti; therefore, we applied a technique in which we used an apico-aortic conduit via posterolateral thoracotomy. We underwent apico-aortic bypass with a hand-made composite graft: $19-\mathrm{mm}$ bioprosthetic valve and a 22-mm woven polyester vascular graft. The surgical intervention successfully decreased pressure gradient across the aortic valve, also separating an incision from chronic infection allowed us to avoid postoperative mediastinitis. The patient had remained in good condition for 15 months without developing any complications.
\end{abstract}

Keywords: aortic stenosis, apico-aortic bypass, apico-aortic conduit, thoracic wall infection

\section{Introduction}

Although aortic valve replacement is the standard surgical procedure for severe aortic stenosis, it carries significant operative risks in patients with circumferentially calcified ascending aorta, left ventricular outflow tract obstruction or history of cardiac surgery, such as coronary artery bypass grafting. ${ }^{1,2)}$ We report a case of 70-year-old woman with severe aortic stenosis and chronic thoracic wall infection due to a previous mastectomy.

Department of Cardiovascular Surgery, Saitama Medical Center, Jichi Medical University, Saitama, Saitama, Japan

Received: August 19, 2011; Accepted: September 29, 2011 Corresponding author: Manabu Shiraishi, MD. Division of Cardiovascular Surgery, Saitama Medical Center, Jichi Medical University, 1-847 Amanuma, Omiya-ku, Saitama, Saitama 3308503, Japan

Email: manabu@omiya.jichi.ac.jp

(C)2011 The Editorial Committee of Annals of Thoracic and Cardiovascular Surgery. All rights reserved.

\section{Case Report}

70-year-old-woman was admitted to our hospital with an exacerbation of congestive heart failure. She had a history of mastectomy and radiation therapy for breast cancer, and was suffering from a chronic thoracic wall infection and radiodermatitis that remained at the surface of the skin. Transthoracic echocardiography upon admission showed severe aortic valve stenosis with heavy calcification, aortic valve area of $0.54 \mathrm{~cm}^{2}$, high aortic valve pressure gradients (mean $53.8 \mathrm{mmHg}$, max $78.0 \mathrm{mmHg}$ ), and pulmonary hypertension. Coronary angiography showed no stenosis in the coronary arteries. Computed tomography (CT) showed severe calcification of the ascending aorta and the aortic arch, and opacity in the right upper lung (Fig. 1). Since median sternotomy was considered to be high risk of postoperative mediastinitis due to chronic infected thoracic wall, we applied a technique using an apico-aortic conduit via posterolateral thoracotomy.

Under general anesthesia, the patient was placed in the left lateral decubitus position. Anesthetic management included a placement of double-lumen endotracheal 


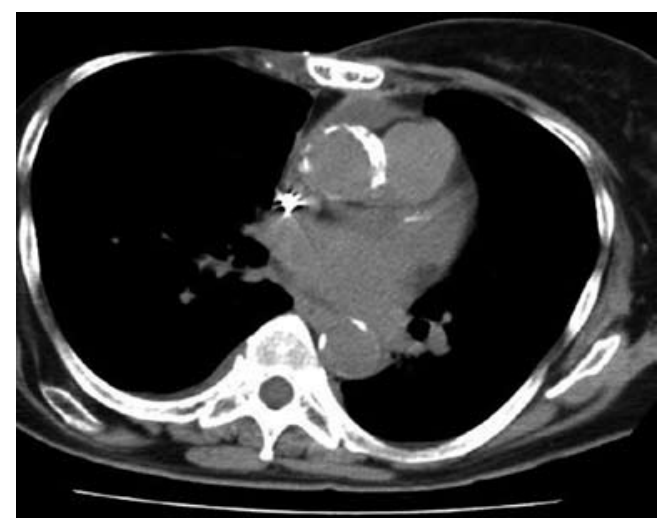

Fig. 1 Computed tomography showed severe calcification of the ascending aorta and loss of the right breast.

tube for single-lung ventilation. The left thoracotomy was performed in the sixth intercostal space. After dividing the inferior pulmonary ligament, we retracted the lung, exposing the cardiac apex and the descending thoracic aorta. The pericardium was then opened, anterior to the phrenic nerve, and the apex was exposed. Under general heparinization, cardiopulmonary bypass (CPB) was instituted by cannulating the right femoral artery, the descending thoracic aorta and the right femoral vein. At first, the descending aorta was total-clamped in two places. A $20-\mathrm{mm}$ woven polyester vascular graft (Hemashield Gold; Boston Scientific, Natick, MA) was sewn to the descending aorta with a 4-0 polypropylene running suture in side-to-end fashion. The patient's body temperature was cooled down to 28 degrees at the commencement of $\mathrm{CPB}$. Under ventricular fibrillation, apical myocardium was punched out with an 18-mm tissue puncher in $2 \mathrm{~cm}$ lateral to the left ventricular apex. A reversed 20-mm woven polyester vascular graft (Hemashield Gold; Boston Scientific, Natick, MA) was passed through the apex into the left ventricle. Twelve interrupted 2-0 nonabsorbable mattress sutures were sewn to anastomose the left ventricular apex and prosthesis. To achieve local hemostasis, we used teflon-felt strips for this anastomosis. After the left ventricular apex and graft were anastomosed, the reversed graft was drawn from the left ventricle (Fig. 2). A hand-made composite graft consisted of a 19-mm bioprosthetic valve (Mosaic; Medtronic, Minneapolis, MN) and a 22-mm woven polyester vascular graft. The distal end of the apical graft was sewn to the composite graft with 4-0 polypropylene running suture. The distal end of the composite graft was sewn to the proximal end of the $20-\mathrm{mm}$ woven polyester vascular graft with 4-0 polypropylene running suture in end-to-end

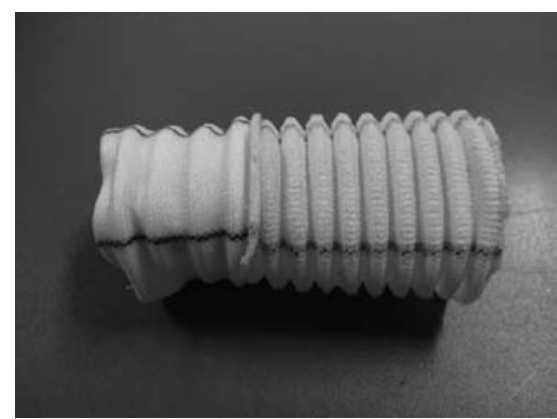

Fig. 2 After a reversed 20-mm woven polyester vascular graft was anastomosed to the left ventricular apex, the reversed graft was drawn from the left ventricle. The reversed part of the graft is in the left ventricular.

fashion. CPB was easily weaned and total We performed $\mathrm{CPB}$ on the patient for 150 minutes and then easily weaned her from it.

Postoperative respiratory status became complicated. Artificial respiration support of more than two weeks was necessary because the apico-aortic conduit and pleural effusion in the left thoracic cavity decreased the left thoracic capacity; however, it was managed successfully. The other postoperative course was uneventful, and no thromboembolic events have been observed. Postoperative echocardiography showed a decreased pressure gradient across the aortic valve (mean $9.3 \mathrm{mmHg}$, max 18.7 $\mathrm{mmHg}$ ) with a fair, left ventricular ejection fraction. Postoperative chest CT demonstrated a conduit between the left ventricular apex and the descending aorta (Fig. 3). The patient has remained in good condition for 15 months without developing any complications.

\section{Discussion}

We presented a case of apico-aortic bypass surgery that was conducted successfully in a patient with high risk of postoperative mediastinitis. The apico-aortic bypass has been demonstrated to be fit in congenital aortic valve stenosis, left ventricular outflow tract anomalies, and acquired aortic valve conditions. ${ }^{3)}$ In our case, due to lack of availability of a rigid right-angled apical connector (Medtronic Hancock Left Ventricular Connector; Medtronic Inc, Minneapolis, MN) in our institution, we utilized a hand-made composite graft. The bypass succeeded in decreasing pressure gradient across the aortic valve, and in avoiding postoperative mediastinitis.

Recently, an alternative to surgical aortic valve replacement in a subset of high-risk patient-transcatheter aortic valve implantation (TAVI)-has emerged. ${ }^{4)}$ This 
treatment can avoid respiratory disorder due to long mechanical ventilation and CPB. In the report of Leon et al, a total of 358 patients with aortic stenosis, who were not considered to be suitable candidates for surgery, underwent randomization at 21 centers. The rate of death from any cause was $30.7 \%$ with TAVI, as compared with $50.7 \%$ with standard surgical therapy, at 1 year. ${ }^{5)}$ Tamburino et al. reported the cumulative incidences of mortality in a total of 663 patients, who underwent TAVI, were $5.4 \%$ at 30 days, $12.2 \%$ at 6 months, and $15.0 \%$ at 1 year. ${ }^{6)}$ These results show that TAVI is beneficial in patients with severe aortic stenosis, who were not suitable candidates for surgery. However, TAVI, as compared with standard therapy, was associated with a higher incidence of a major stroke, atrioventricular conduction system injury and major vascular complications. Furthermore, procedural complications were strongly associated with early mortality. ${ }^{5,6)}$ Due to the higher incidence of complications and lack of generality of TAVI in Japan, apico-aortic bypass can be an option for the treatment of aortic stenosis when there is a high risk of stroke or comorbidities.

Renzulli et al. reported long-term results of apico-aortic valved conduit. In their report, despite the high incidence of reoperation due to conduit valve failure, apicoaortic conduit implantation produced good hemodynamic outcome and improved the quality of life in patients. ${ }^{7)}$ The least invasive strategy would include on-pump beating apico-aortic conduit under normothermia ${ }^{1)}$ and offpump apico-aortic conduit insertion with a rigid apical connector, ${ }^{8)}$ which avoids myocardial ischemia in high risk patients and hemorrhage from an abnormality of hemostasis under hypothermia. In the near future, these technical modifications may become mandatory to reduce surgical risk.

\section{Conclusion}

We report a case of an apico-aortic bioprothesis-valved conduit for severe aortic stenosis and chronic thoracic wall infection. When a conventional approach is not available, technical modifications are important to reduce surgical risk.

\section{Disclosure Statement}

No external funding was obtained for the work presented here. The authors have no conflicts of interest to declare.

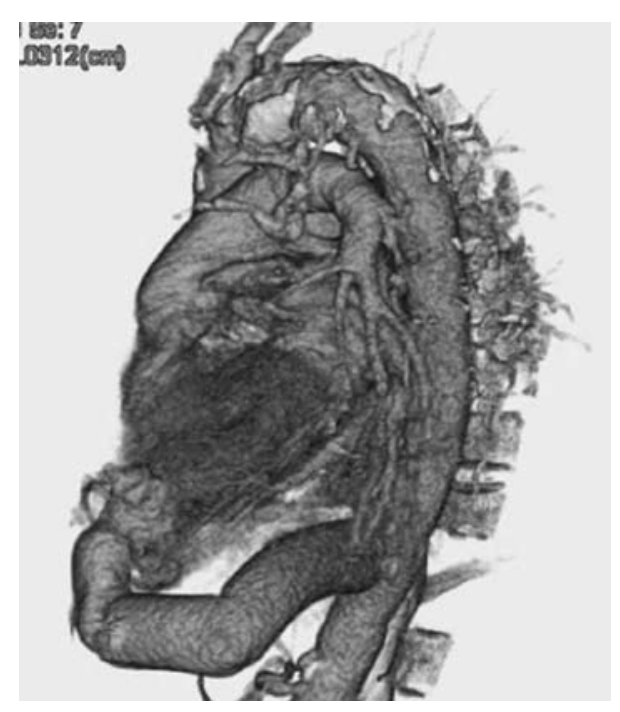

Fig. 3 Image reconstruction from a multi-detector computerized tomography showed the conduit between the left ventricular apex and the descending aorta.

\section{References}

1) Hirota M, Oi M, Omoto T, et al. Apico-aortic conduit for aortic stenosis with a porcelain aorta; technical modification for apical outflow. Interact Cardiovasc Thorac Surg 2009; 9: 703-5. Epub 2009 Jul 7.

2) Takahashi Y, Tsutsumi Y, Monta O, et al. Thrombus formation due to flow competition after apico-aortic conduit. Eur J Cardiothorac Surg 2010; 37: 978-9. Epub 2009 Oct 27.

3) Sadaba R, Alvarez L, Juaristi A, et al. An apico-aortic conduit in a case of patient-prosthesis mismatch. Rev Esp Cardiol 2009; 62: 1194-5.

4) Walther T, Simon P, Dewey T, et al. Transapical minimally invasive aortic valve implantation: multicenter experience. Circulation 2007; 116: I240-5.

5) Leon MB, Smith CR, Mack M, et al. Transcatheter aortic-valve implantation for aortic stenosis in patients who cannot undergo surgery. N Engl J Med 2010; 363: 1597-607. Epub 2010 Sep 22.

6) Tamburino C, Capodanno D, Ramondo A, et al. Incidence and predictors of early and late mortality after transcatheter aortic valve implantation in 663 patients with severe aortic stenosis. Circulation 2011; 123: 299308. Epub 2011 Jan 10.

7) Renzulli A, Gregorio R, De Feo M, et al. Long-term results of apico-aortic valved conduit for severe idiopathic hypertrophic subaortic stenosis. Tex Heart Inst J 2000; 27: 24-8.

8) Chahine JH, El-Rassi I, Jebara V. Apico-aortic valved conduit as an alternative for aortic valve re-replacement in severe prosthesis-patient mismatch. Interact Cardiovasc Thorac Surg 2009; 9: 680-2. Epub 2009 Jul 30 . 